\title{
Characterization of a Linear Streak Artifact With Pulse Inversion Tissue Harmonics in Musculoskeletal Sonography
}

\author{
Aya Kamaya, MD, Sarah Abate, BS, Bin Nan, PhD, \\ Inderpreet Grover, Ronald S. Adler, MD, PhD, \\ David Jamadar, MBBS, Jonathan M. Rubin, MD, PhD
}

Objective. To understand a linear artifact that projects deep to reflective structures that move rapidly while using tissue harmonic imaging with pulse inversion (PI) sonography. We hypothesize that this artifact is due to a cancellation error between firings in PI imaging, and it is, therefore, similar in generation to the twinkling artifact in color Doppler sonography. This artifact could be studied with the use of surfaces of different roughness to represent different rates of motion, in which roughness corresponds to spatial fluctuations in surface height. Given very slight variations in beam focusing as occurs with sonographic imaging arrays, these spatial fluctuations translate into temporal fluctuations in the received signal as would occur with tissue motion. Methods. We scanned 4 different sandpaper grits and a smooth surface through a water path using fundamental and PI mode, 1- and 2-pulse techniques, respectively. The sandpaper and the smooth surface were scanned through a water path at mechanical indices of 0.1 to 0.7 . Four independent images were subtracted pairwise to remove nonfluctuating signals. These noise pixels were counted and analyzed. Results. Analysis of variance showed that the noise generated behind the different surfaces was highly significantly different. Two-tailed $t$ tests generally showed significant differences in the quantity of noise between fundamental and harmonic imaging behind the roughest 3 grades of sandpaper. A multiple regression model showed significantly greater slopes for harmonic imaging for all grades of sandpaper and the smooth surface. Conclusions. The noise and, by extension, the linear streak artifact in musculoskeletal imaging are dependent on the mechanical index and are functions of sandpaper roughness. This would be equivalent to a subtraction error between 2 firings due to soft tissue motion, and the artifact may be a way to identify rapid soft tissue motion in Pl images. Key words: musculoskeletal sonography; pulse inversion; sonographic artifacts; tissue harmonic imaging.

\begin{abstract}
Abbreviations
$\mathrm{MI}$, mechanical index; PI, pulse inversion; SOAB, soundabsorbing

Received June 24, 2004, from the Departments of Radiology (A.K., S.A., I.G., D.J., J.M.R.) and Biostatistics (B.N.), University of Michigan, Ann Arbor, Michigan USA; and Department of Radiology, Hospital of Special Surgery, New York, New York USA (R.S.A.). Revision requested July 13, 2004. Revised manuscript accepted for publication August 17, 2004.

This paper was the recipient of the 2003 Larry Mack Research Award from the Society of Radiologists in Ultrasound and was presented at the 13th Annual Meeting of the Society of Radiologists in Ultrasound, Chicago, Illinois, October 2003.

Address correspondence and reprint requests to Jonathan M. Rubin, MD, PhD, Department of Radiology, B1D502, 1500 E Medical Center Dr, Ann Arbor, MI 48109-0030 USA.

E-mail: jrubin@umich.edu.
\end{abstract}

7 issue harmonic imaging is a relatively new mode of sonographic imaging. Images are formed by using the harmonic signals that are generated by tissue while filtering out reflected fundamental echo signals that are generated by the transmitted acoustic energy. ${ }^{1}$ The beam characteristics of tissue harmonic imaging are such that clutter noise from side lobes and reverberations are suppressed considerably. In a standard implementation of tissue harmonic imaging, 2 sequential pulses with a $180^{\circ}$ phase shift are produced in rapid succession by the transducer. When the sound beam is reflected back to the transducer, the reflected signals from each pulse are summed. The fundamental echoes perfectly cancel, whereas harmonic signals remain in phase and add coherently. This is often referred 
to as pulse inversion (PI) imaging. By removal of the fundamental echoes and their associated noise, the sonographic image quality is improved. To date, there is little to no literature on sonographic artifacts seen on harmonic imaging.

We describe and characterize a transient linear artifact seen on PI sonography that projects at the margins of bones, tendons, nerves, and other solid objects when these structures are induced to move rapidly across an ultrasonic field through provocative maneuvers (snapping) during tissue harmonic imaging (Figure 1). (Investigational Review Board approval was waived for this single clinical example.) At times, this movement occurs so rapidly that the actual motion of the tendon out of its normal location may be difficult to perceive. Generation of this artifact may help in identification of this abnormal movement.

Given that this artifact appears to be dependent on the rate of motion, we sought to simulate this artifact by using surfaces of different roughness to create the motion artifact. The rougher the surface, the higher the amplitude and the more rapid the spatial fluctuations of the surface height around a mean position. ${ }^{2}$ These spatial fluctuations could be analogous to motion in time if the beam position fluctuates temporally, no matter how slight this fluctuation may be. In this regard, we hypothesize that because PI is a 2pulse technique, the artifact might be analogous to the twinkling artifact in color Doppler sonography. ${ }^{3}$ We therefore decided to scan different grades of sandpaper and a smooth surface to try to demonstrate and understand the linear streak artifact in PI harmonic imaging.

\section{Materials and Methods}

All scanning was performed using an HDI 5000 sonography machine (Philips Medical Systems, Bothell, WA) with an L7-4 linear array probe fixed in a ring clamp (Figure 2). We used this transducer and machine because the shape and size of the transducer made it easy to hold in a ring clamp. We were also able to systematically change and observe the mechanical index (MI) in the gray scale mode. Finally, we knew that harmonic imaging in the HDI 5000 machine used a PI technique (information courtesy of J. Powers, Philips Medical Systems), which was required for our experiments. (A single-pulse technique in which the return signal is filtered to remove the funda-
Figure 1. Snapping left ulnar nerve in a 47-year-old male subject. A, Transverse view of the flexed elbow with the ulnar nerve (arrow) lying in an abnormal position anterior to the medial epicondyle (E). B, Transverse view of the ulnar nerve (arrows) as it snaps back into the ulnar groove, posterior to the medial epicondyle, creating a streak artifact (open arrows). Note that the streak artifact obscures visualization of the central portion of the nerve. C, Transverse view of the ulnar nerve (arrow) at rest in the extended elbow, lying in its anatomic location posterior to the medial epicondyle. $\mathrm{F}$ indicates common flexor origin; and T, triceps.

A

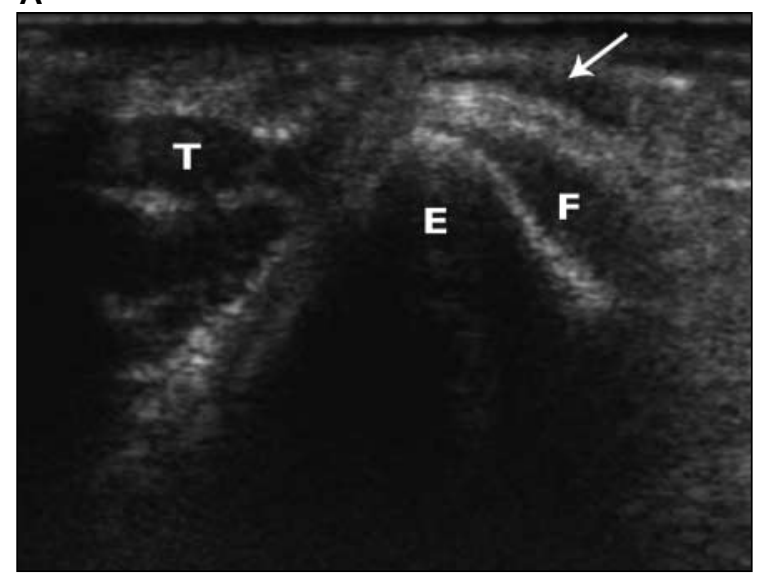

B

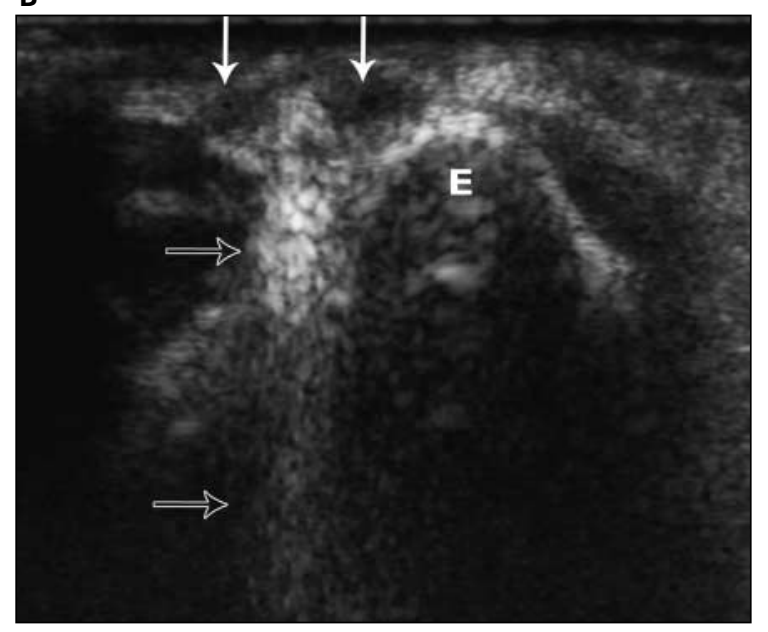

C

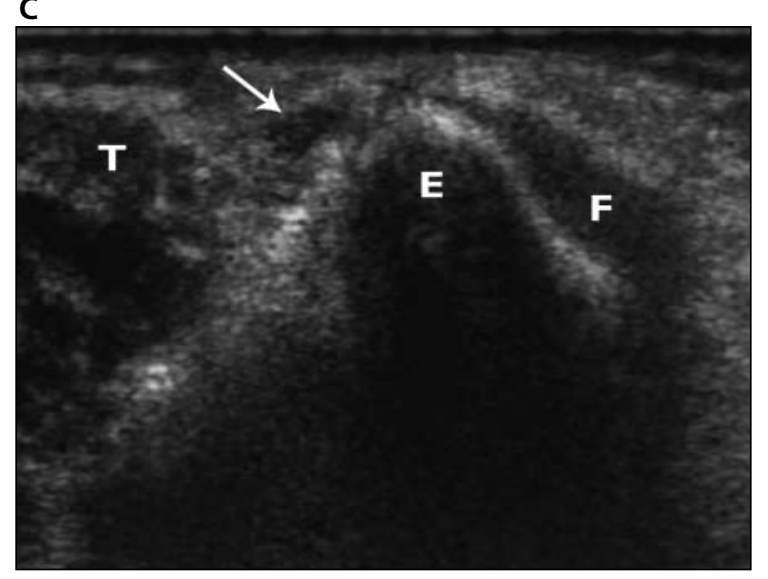


mental signal, which is an alternative mode of harmonic imaging, would presumably not produce this artifact.) Holding the transducer in a ring clamp ensured that the transducer was stationary and, therefore, did not affect or contribute to the linear streak artifact because of physical motion relative to the reflecting surfaces. Sandpaper roughness is quantified by the grit level. The lower grit levels are rougher or courser, whereas the higher grit levels are finer. Four different sandpaper grits were scanned in a water bath: $80,220,320$, and 600 grit, with 80 grit being the roughest sample and 600 grit being the least rough. A flat sound-absorbing (SOAB) rubber surface was also scanned, which served as a smooth control. Objects were scanned at a distance of approximately $2.0 \mathrm{~cm}$ from the transducer face.

Gray scale gain on the HDI 5000 machine is controlled by a knob with 42 possible positions as well as a set of slide pods for the time-gain compensation at each depth position. To ensure

Figure 2. L7-4 linear array probe fixed in a ring clamp. The probe is in a basin used to hold water for scanning.

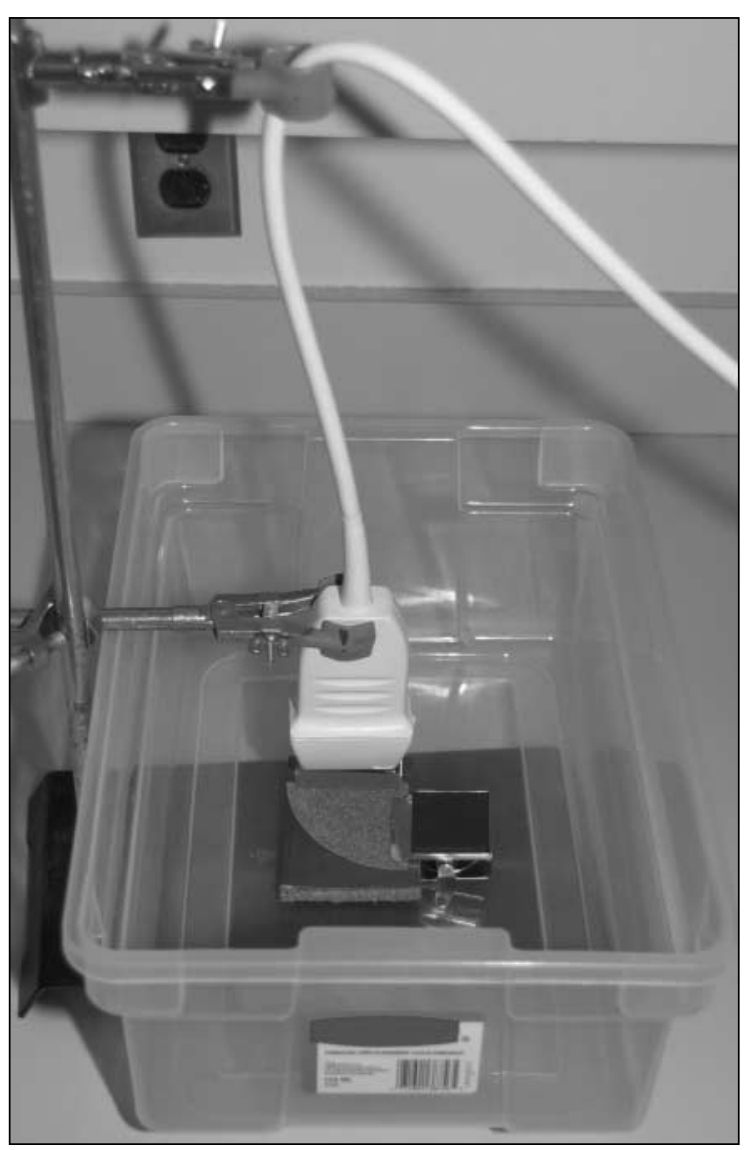

consistency, the knob was set at the level just above the point at which, if the knob were turned to the left, the time-gain compensation curve would begin to curve leftward. The time-gain compensator slide pods were set such that, between 1 and $3 \mathrm{~cm}$ deep from the scan head surface, the levers were at maximum, and above and below these levels, the levers were at minimum. These adjustments were possible because our targets were very strong reflectors, and there was essentially no overlying attenuation in the water bath. These lever settings decreased the amount of reverberation signal behind the sandpaper and maximized the signal from the level of the sandpaper and the immediately adjacent artifact.

Sandpaper was securely clipped to the flat $\mathrm{SOAB}$ rubber surface (Figure 3). This further decreased the amount of reverberation artifact seen behind the layer of sandpaper and ensured that the object was stationary and not affected by ultrasonic radiation force.

Each different surface was scanned in the harmonic PI mode and in the fundamental mode. We used the fundamental mode as a single-pulse technique for comparison. As mentioned above, the HDI 5000 machine does not have a singlepulse harmonic mode. The MI values were systemically increased from 0.1 to 0.7 by 0.1 increments. When the machine did not allow an exact increment of 0.1 , the MI was set at the closest setting the machine would allow, which was generally \pm 0.01 MI from the desired 0.1 incre-

Figure 3. Sandpaper firmly clipped to a dark gray SOAB rubber surface.

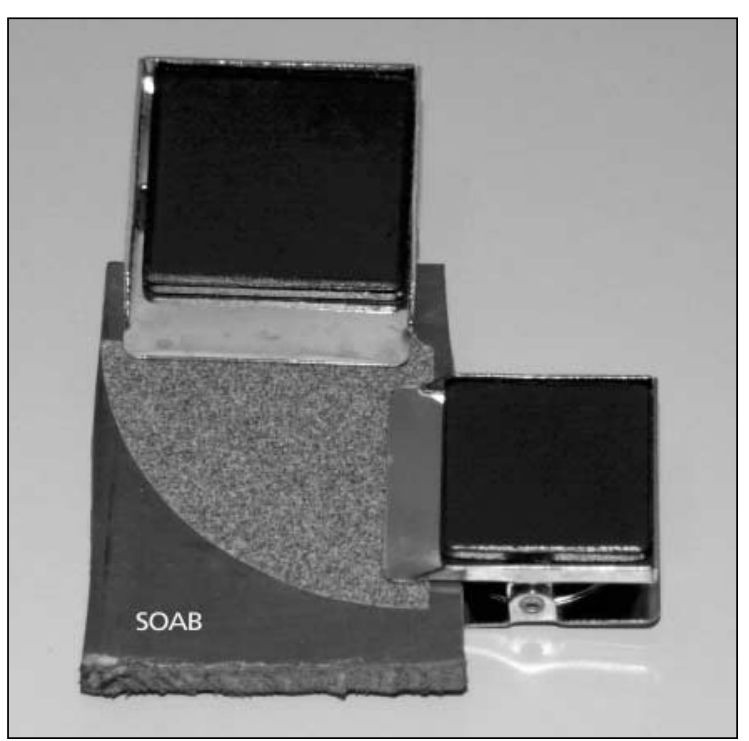


ment. At each setting, sequential frames were stored in a cine loop. Images at frames 20, 40, 60, and 80 were then captured and saved to ensure that there was no bias in selecting images.

Images were saved in bit-map format and were transferred to Photoshop (Adobe Systems, San Jose, CA). Images were converted to the blackand-white mode with 256 gray levels mapped numerically from 0 to 255 . Each image was completely selected (select all) then pasted into separate layers within the same file. This ensured that the images were perfectly superimposed. All layers were cropped simultaneously at the same location and saved as a single file. This ensured that each image was cropped at exactly the same location and that the size of the crop box was exactly the same. The size of the crop box was chosen to encompass the image from the $1-\mathrm{cm}$ depth mark to the 3-cm depth mark and to encompass the entire scan surface of the image. When scanning in the harmonics mode, the scale was slightly altered by the machine, which was a consistent finding. To account for this, the same crop box size was used, but the upper level of the box was justified with the 1-cm depth mark and then shifted to encompass the entire image transversely.

Each layer was subtracted pairwise in Photoshop to remove any nonfluctuating signals. This was obtained by having the calculations command blend 2 individual layers by using the subtract option, with the results placed in a new document (Figure 4). This operation in effect subtracted 1 image from the other, producing 6 independent samples at each setting for analysis. Each sample was saved as a separate file. A histogram of the residual noise in each image was generated by the program on the basis of the gray scale map. The mean, SD, median, and pixel count were recorded on a spreadsheet. The histogram represented only the magnitude of the differences between samples; therefore, no negative values were displayed. To differentiate the background noise from the highly fluctuating noise generated from the surface being evaluated, a histogram was generated in Photoshop on a fixed area $(1 \times 0.5$ in $[2.5 \times 1.25 \mathrm{~cm}])$ in the top left corner of each sample. This region fell entirely within the water path overlying the reflecting surface. The background noise was selected by clicking on the top left corner of the sample with the magic wand tool with a tolerance of 5 pixels. This means that all subtracted pixel pairs having gray scale differences of 5 or less were not counted in the overall noise pixel count for a given image. The tolerance of 5 always defined an upperbound threshold of at least 10 SDs beyond the mean difference for the noise in the water path. Hence, by removing all values of 5 or less, random or electronic noise would generally be removed from the subtracted pair of images being analyzed. By taking the inverse of this selection in Photoshop, the result would be almost completely due to the noise produced by the surface being scanned, corresponding to the

Figure 4. Fluctuating flamelike projections shown extending below the sandpaper surface when 80-grit sandpaper is scanned with harmonic Pl imaging. A, Layer 3: first image obtained from a cine loop with arrows pointing at 2 flamelike projections. B, Layer 2: second image of the same sandpaper obtained from a different time position in the cine loop. C, Result of the subtraction in a new document. A few visible dots represent the fluctuating or nonsubtracted noise between the 2 images.

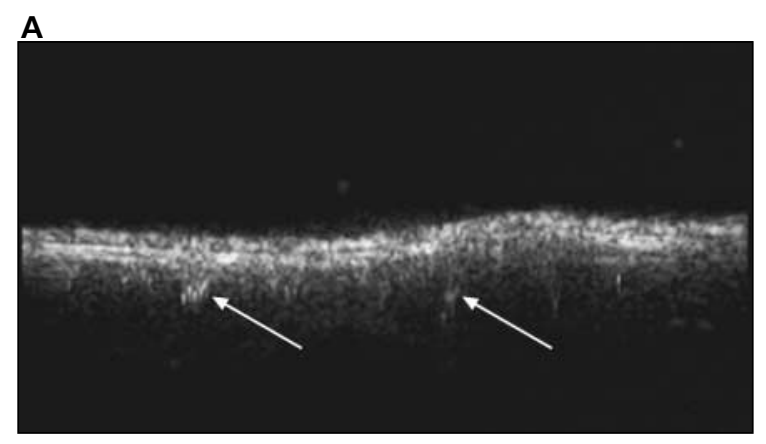

B

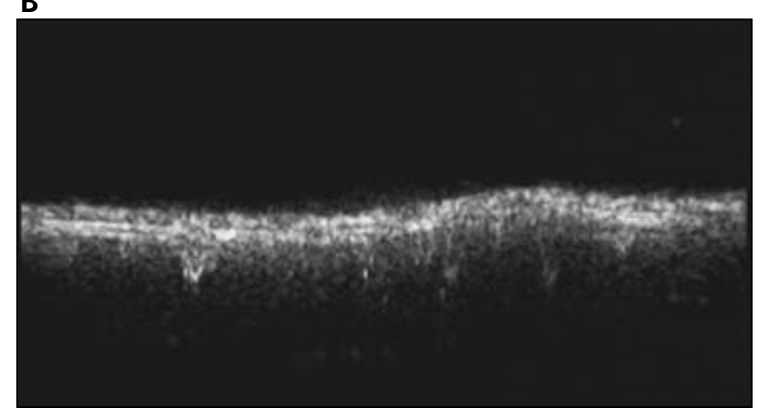

C

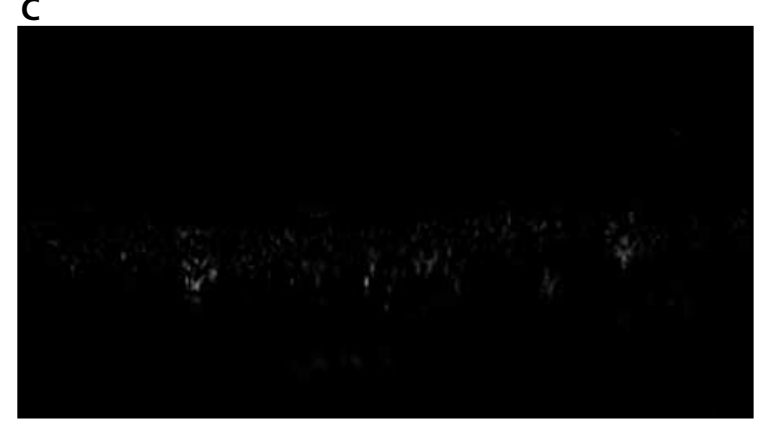


presumed cancellation artifact (Figure 5). A histogram was generated on this selection, and the mean, SD, median, and pixel count were recorded on a spreadsheet (Figure 6).

Because our data consisted of absolute pixelto-pixel differences between pairs of images, there were no negative values. Therefore, the data set was not gaussian distributed. To make the data more "normal" and to decrease the overall variance of the data, we base 10 log transformed on the data before analysis after adding 1 to each of the noise values; 1 was added to remove any zeros in the original nontransformed data set. Analysis of the log-transformed data then included a 1-way analysis of variance to detect whether there was any difference among the noise components for the different surfaces on PI and fundamental imaging. $P<.05$ was taken as proof that there was a difference among the groups. If a difference was detected, we then compared the total quantity of noise generated in the harmonic mode versus the fundamental mode for each surface by using a 2-tailed $t$ test assuming unequal variances between the fundamental and harmonic data. We made this assumption of unequal variances on the basis of the fact that the harmonic data themselves constitute a subtraction technique, meaning that they are doubly subtracted in our analysis. Subtraction will double the variance of like-distributed independent random variables. The subtraction is performed by the harmonic detection itself when it adds the 2 separate $180^{\circ}$ outof-phase firings to produce an image. Given this addition of $180^{\circ}$ out-of-phase firings, which corresponds to a subtraction, we would a priori suspect that the harmonic imaging would have a greater variance than the fundamental imaging. We performed the $t$ test after log transforming the data as described above. Data are presented as means and SDs of the log-transformed data. Again, differences with $P<.05$ were taken as significant (Table 1).

In addition, we also determined whether there was any possible functional relationship between roughness and MI by using linear regression. A functional relationship was considered present if the $95 \%$ confidence limits of the slopes of regression of the log-compressed data did not include 0 (Table 2). If the limits included 0 , we assumed that the slope of regression was not significantly different from 0 , and, therefore, no functional relationship between roughness of

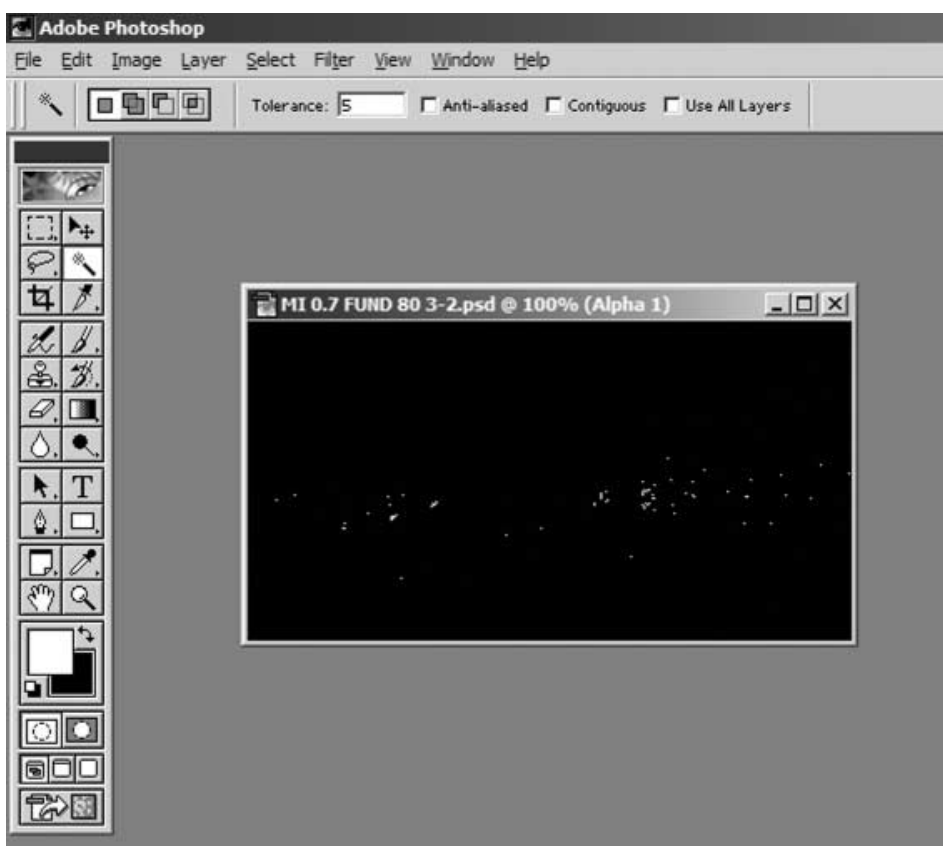

Figure 5. Selected pixels corresponding to the presumed cancellation artifact.

the given grade and MI could be assumed. We also compared the noise relationship of PI harmonic versus fundamental imaging for each surface. This was based on the following model:

(1) $\log (N+1)=b_{0}+b_{1} M I+b_{2} X+b_{3}(M I \times X)+\varepsilon$,

where $N$ was the noise value for each MI measurement; $X$ was an interaction term; and $\varepsilon$ was measurement error. In this model, $X=0$ for fun-

Figure 6. Histogram generated from Figure 5.

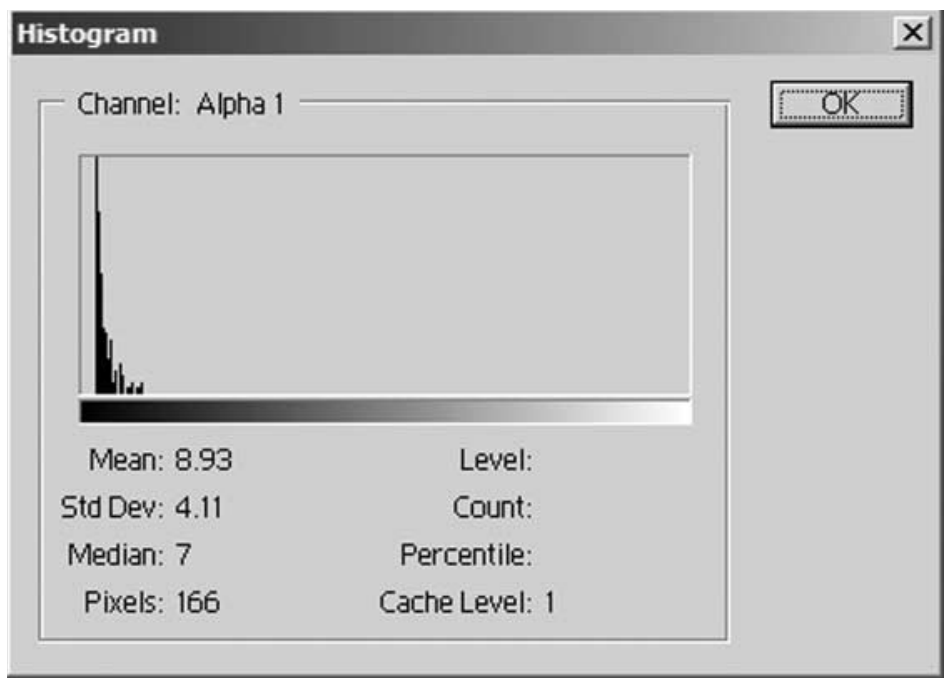


Table 1. Log-Transformed Numbers of Unsubtracted Pixels Behind Surfaces of 4 Sandpaper Grits and an SOAB Surface in Fundamental and Harmonic Modes

\begin{tabular}{lccl}
\hline Surface & $\begin{array}{c}\text { Log Fundamental } \\
\text { (Pixel No.) }\end{array}$ & $\begin{array}{c}\text { Log Harmonic } \\
\text { (Pixel No.) }\end{array}$ & Comparison, $\boldsymbol{P}$ \\
\hline 80 grit & $2.282 \pm 0.320$ & $2.790 \pm 0.665$ & $<.00004$ \\
220 grit & $1.867 \pm 0.696$ & $2.616 \pm 0.716$ & $<.000006$ \\
320 grit & $1.022 \pm 0.371$ & $1.324 \pm 0.694$ & $<.016$ \\
600 grit & $1.886 \pm 0.574$ & $1.949 \pm 0.791$ & $<.678$ \\
SOAB & $0.721 \pm 0.484$ & $0.841 \pm 0.586$ & $<.311$ \\
\hline
\end{tabular}

The measurements were the averages of pairwise subtractions of independent measurements of identical regions made from MI values of 0.1 to 0.7 , with each cell representing 42 measurements. Variations are means \pm SDs of log-transformed values.

damental imaging data, and $X=1$ for harmonic imaging data. The coefficient of interest was $b_{3}$, which represented the difference in slopes between fundamental and harmonic imaging for each surface. $P<.05$ in this multiple regression model for $b_{3}$ was taken as a significant difference between harmonic and fundamental imaging for a given surface as a function of MI (Table 3).

\section{Results}

Subjectively, linear streak artifacts were observed only on harmonic imaging, consistently and most strongly behind sandpaper and at higher MI levels. These artifacts appeared as fluctuating flamelike projections extending down from the sandpaper surface (Figure 4). With an increase in

Table 2. Slopes of Linear Regression Lines Drawn Through the Logarithms of the Numbers of Unsubtracted Pixels for Each Sandpaper Surface and the SOAB Surface as a Function of MI

\begin{tabular}{lccc}
\hline & $\begin{array}{c}\text { Slope, Log } \\
\text { Pixels/MI }\end{array}$ & \multicolumn{2}{c}{ 95\% Cl } \\
\cline { 3 - 4 } Surface & 0.14 & Log Lower & Log Upper \\
\hline Fundamental 80 grit & 1.01 & -0.37 & 0.64 \\
Fundamental 220 grit & 0.86 & 0.23 & 2.11 \\
Fundamental 320 grit & 1.63 & 0.85 & 2.41 \\
Fundamental 600 grit & -0.034 & -1.2 & 0.52 \\
Fundamental SOAB & 2.5 & 1.81 & 3.19 \\
Harmonic 80 grit & 3.24 & 2.74 & 3.72 \\
Harmonic 220 grit & 2.14 & 1.23 & 3.04 \\
Harmonic 320 grit & 3.41 & 2.73 & 4.08 \\
Harmonic 600 grit & &
\end{tabular}

The $95 \%$ confidence intervals $(\mathrm{Cl})$ around these slopes are shown as well. If the $95 \%$ confidence intervals contain 0 slope, then it is assumed that there is no functional relationship between the amount of noise present and the $\mathrm{MI}$. There is a functional relationship in the harmonic mode between the $\mathrm{MI}$ and cancellation noise for all of the surfaces studied. the MI, these flames became more obvious. No linear flamelike artifacts were subjectively observed on fundamental imaging (Figure 7) or on the smooth, flat surface (SOAB).

Quantitatively, results of the analysis of variance of the data were highly significant $(P<<.001)$. It was therefore deemed appropriate to perform more indepth analyses of the data. Comparisons between the amount of noise behind the surfaces in fundamental and harmonic modes correlated well with roughness (Table 1). The 2 rougher sandpapers, 80 and 220 grit, had highly significant differences in the levels of noise between the subtracted fundamental and subtracted harmonic images, whereas the noise in the harmonic subtracted images was significantly greater than in the fundamental images with the 320-grit sandpaper. However, the level of significance was orders of magnitude less for the 320-grit sandpaper compared with the 80- and 220-grit sandpapers. There was no significant difference between the levels of harmonic and fundamental noise for 600-grit sandpaper and the smooth SOAB surface.

Regression analysis showed true functional relationships between the amounts of noise and MI for all surfaces tested in the harmonic mode. We also saw functional relationships in the fundamental mode for the 320- and 600-grit sandpaper surfaces. This was based on the $95 \%$ confidence limits of the regression slopes not including 0 (Table 2). Multiple regression analysis, however, showed that the noise as a function of MI was significantly greater for PI harmonic imaging than for fundamental imaging for all surfaces (Table 3 ).

Table 3. Comparisons of Linear Regression Slopes Between Fundamental and PI Harmonics

\begin{tabular}{lll}
\hline Surface & \multicolumn{1}{c}{$\boldsymbol{b}_{\mathbf{3}}$} & \multicolumn{1}{c}{$\boldsymbol{P}$} \\
\hline 80 grit & 2.354 & .0000003 \\
220 grit & 2.265 & .00017 \\
320 grit & 1.298 & .013 \\
600 grit & 1.725 & .000781 \\
SOAB & 2.2 & .00004 \\
\hline
\end{tabular}

There is 1 multiparameter regression for each surface studied; the regressions are taken over the MI values from 0.1 to 0.7 ; and the analysis includes both fundamental and PI harmonic data together. The comparison is based on the value of the coefficient of the interaction term in the regression model (Equation 1). $P<.05$ for this coefficient suggests that the slopes are significantly different. 


\section{Discussion}

Many different tendons have been reported ${ }^{4-9}$ to manifest clinically with snapping, including the iliopsoas tendon and the iliotibial band, the biceps femoris tendon, the semitendinosus tendon, and the brachialis tendon. Sonography is very useful for imaging these structures dynamically.

In this regard, we have now identified a linear streak artifact in harmonic imaging at the margins of bony or tendinous structures when these structures are induced to move rapidly through provocative maneuvers (Figure 1). The artifact appears as a linear, bright reflection or band extending deep to the surface of the moving nerve or tendon. It has only been seen in tissue harmonic imaging modes in which PI has been used to suppress the fundamental signal. This suppression relies on perfect alignment of the tissue between the 2 firings in the PI mode. If there is motion that is sufficiently rapid between the 2 firings so that this alignment is lost, subtraction of the fundamental signal will be incomplete. In fact, if the displacement is equivalent to half a wavelength, the returning signal will have twice the amplitude of the fundamental signal. This is a huge signal relative to the typical harmonic signal, which is on the order of $18 \mathrm{~dB}$ down from the fundamental signal. Such a strong signal could definitely appear as an artifact. Furthermore, multiple reverberations of this strong signal would also be strong relative to the harmonic signal. These reverberations could extend the artifact deep to the reflecting boundary, which is what is seen in the linear streak artifact.

We hypothesized that scanning a highly reflective rough surface such as sandpaper with harmonic imaging would simulate this artifact. This was based on the notion that PI harmonics and color or power Doppler imaging are similar in that they are all multiple-pulse techniques. Given that, the linear streak artifact can be thought of as a gray scale analogue of the wellknown twinkling artifact in color Doppler sonography. ${ }^{3}$ This artifact is generated at the surface of highly reflective, rough objects and is likely due to very small errors in phasing of the array elements in a ultrasonic scan head. Because beam focusing and steering depend on very precise phase delays between the piezoelectric elements in a scan head, any variation will very slightly

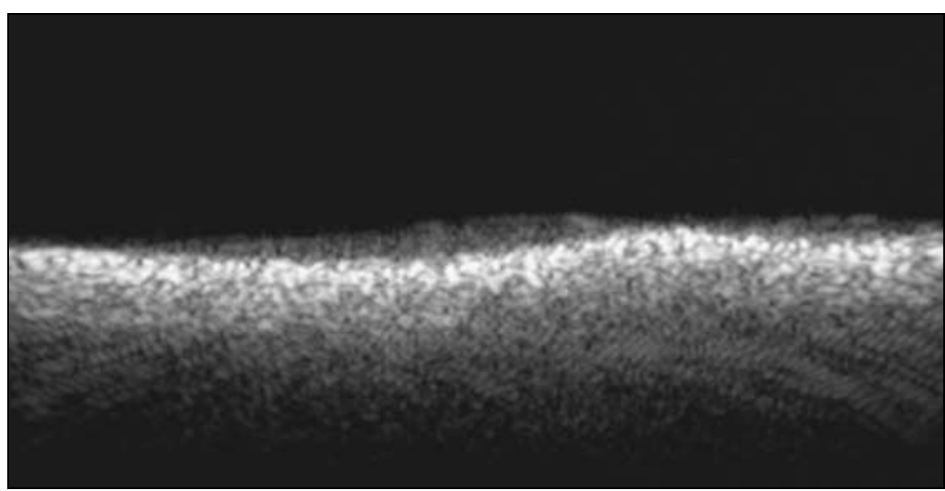

Figure 7. No flamelike projections are shown below the surface when 80-grit sandpaper is scanned in the fundamental mode. When images from a cine loop are subtracted pairwise, few if any pixels remain, indicating much less fluctuation between images than shown on harmonic imaging.

change the direction of the ultrasound beam. These fluctuations are very small, on the order of $10^{-30}$, and they must be small, or no imaging would be possible. However, given a rough, highly reflective surface, these slight errors will create path length differences that will look like motion in Doppler modes. We think that very similar effects might occur in PI harmonics.

In our case, phase jitter created very slight fluctuations in the direction and focusing of the ultrasound beam, which, because of path length differences, produced inaccurate firing-to-firing subtractions, which produced the artifacts. In vivo, the tissue motion itself is dominating, presenting different reflectors to the ultrasound beam from firing to firing. These will not appropriately subtract, leaving a strong residual fundamental signal, the linear streak artifact. This is analogous to a strategy that has been used in gray scale blood flow imaging. ${ }^{10}$ The signal differences detected with soft tissue motion are, of course, orders of magnitude stronger than those produced from red blood cells.

If this hypothesis is correct, rougher surfaces should have relatively more artifacts than smoother surfaces. The rougher the surface, the higher the root mean squared fluctuation in the surface height, which corresponds to a more rapid spatial variation in the surface being insonated. To an ultrasound beam with any temporally dependent spatial fluctuations, this will look like a moving target, with greater roughness corresponding to greater apparent motion. This is what we saw in general. The amount of the total artifact was significantly greater behind the 
rougher sandpaper grits, 80,220 , and 320 grit, in the harmonic mode than in the fundamental mode. In fact, the differences between harmonic and fundamental noise levels were much more significant with 80 and 220 grit than with 320 grit. More interestingly, there was no significant difference between the 2 imaging modes with the smoother surfaces, 600 grit and SOAB. This is consistent with the statement the small amounts of variation may not generate the artifact.

These findings would also explain why such artifacts are not routinely seen with other moving structures such as bowel peristalsis. This is because the phase error must be large enough to permit a substantial fundamental signal to bleed through. If the firing interval is short relative to the motion, the phase errors will be very small, similar to smooth surfaces, and little or no artifact will be visible. However, one could devise firing schemes to enhance this error, that is, relatively slow pulse repetition intervals. Such methods could be quite sensitive to motion but would also be sensitive to minor soft tissue motions as well. Such soft tissue artifacts can often be seen in gray scale flow imaging techniques $^{10}$ and amplitude color flow techniques such as power Doppler imaging. ${ }^{11}$

We did see a trend in the amount of noise behind different surfaces with increasing MI based on linear regression analysis in the harmonic mode for all surfaces, even SOAB. This is not surprising given that any phase jitter will produce an error that will produce artifacts independent of surface roughness, although roughness will magnify this error. Given that, a trend of increasing visibility of the noise with MI could be expected with PI harmonic imaging. This is because the subtraction noise artifacts appear stronger when the signal is stronger. For any given phase error, the difference in magnitude between any 2 signals is greater when the primary signals are stronger. For instance, suppose a given phase error produces a maximal difference signal of $6 \mathrm{~dB}$ at a particular MI setting. If the MI is then increased by a factor of 3 for a constant carrier frequency, the maximal difference signal will increase to $15 \mathrm{~dB}$. This will obviously be easier to see. Also, all reverberations will be proportionately increased, making the flamelike artifacts more visible. Hence, a trend with increasing MI is to be expected in harmonics. This trend was present for all our surfaces and was significantly greater than for fundamental imaging. In addition, given this trend, the fact that our measurements did not detect differences between the quantities of subtraction noise behind 600-grit and SOAB surfaces for PI harmonics and fundamental imaging might only have been due to the fact that we could not test outputs higher than $0.7 \mathrm{MI}$. If we could have, perhaps all our surfaces would have manifested total noise differences. Even if these differences did appear, the subtraction noise would still be significantly greater for PI harmonics than fundamental imaging in all cases on the basis of the significantly greater slopes with regression for PI harmonics for all surfaces (Table 3 ).

The fact that a trend was seen with 320 - and 600grit sandpaper only in fundamental imaging likely has the same explanation; that is, if we could have gone to higher MIs, we might have seen positive trends with all our samples. Phase jitter occurs in fundamental imaging as well as in harmonics, and we are still subtracting different frames in a cine loop. This could manifest as a trend with a more demonstrable difference with higher MIs, just as in harmonics, although the amount of detectable noise should be smaller, as we have seen. Furthermore, the huge increase in a signal that one would see for subtraction errors in PI harmonics, in which phase errors can produce fundamental signals that are on the order of $18 \mathrm{~dB}$ stronger than the harmonics, would not occur for fundamental imaging in our experiments. Finally, the subtraction noise in fundamental imaging is only a construct of our experiments. Unlike in PI harmonics, this will not occur in clinical imaging. Fundamental transmissions are not subtracted in clinical imaging, and phase errors due to motion will not be a factor. Hence, the artifact will not be seen in fundamental imaging.

Finally, note that the absolute numbers of noise pixels are not totally consistent throughout the data in Table 1 . The numbers of noise pixels for 600-grit sandpaper are greater in both fundamental and harmonic imaging modes than those for 320-grit sandpaper. Although we used the same settings throughout, such an event is not a total surprise because we were looking at a noise signal, which obviously could vary. In addition, the 600-grit paper was made of a different substance than the 320-grit paper. The important comparison, however, is between the harmonic and fundamental images for any given grit, and here the relationship between single- and multiple-firing modes holds up. 
In conclusion, our experiments show an increase in the subtraction noise behind sandpaper surfaces as a function of roughness and MI. Given the effects of phase jitter as a cause of very slight variations in focusing of ultrasonic transducer arrays, this noise would be a consequence of errors in subtraction between firings that are designed to remove the fundamental signal in PI harmonic imaging. Using a hypothesis that degrees of roughness in our experiments correspond to different rates of motion in soft tissues, our experiments suggest that the linear artifact projecting from the margins of rapidly moving tendons in harmonic imaging in musculoskeletal sonography is produced by a phase cancellation error between the 2 firings in the PI mode. The artifact is analogous to the twinkling artifact in color Doppler imaging. It is possible that this artifact could prove useful clinically to document rapid soft tissue motion that might be difficult to document by other means.

\section{References}

1. Tranquart F, Grenier N, Eder V, Pourcelot L. Clinical use of ultrasound tissue harmonic imaging. Ultrasound Med Biol 1999; 25:889-894.

2. Chiang EH, Laing TJ, Meyer CR, Boes JL, Rubin JM, Adler RS. Ultrasonic characterization of in vitro osteoarthritis articular cartilage with validation by confocal microscopy. Ultrasound Med. Biol 1997; 23:205-213.

3. Kamaya A, Tuthill TA, Rubin JM. Twinkling artifact on color Doppler sonography: dependence on machine parameters and underlying cause. AJR Am J Roentgenol 2003; 180:215-222.

4. Pelsser V, Cardinal E, Hobden R, Aubin B, Lafortune M. Extraarticular snapping hip: sonographic findings. AJR Am J Roentgenol 2001; 176:67-73.

5. Wunderbaldinger $\mathrm{P}$, Bremer C, Matuszewski L, Marten K, Turetschek K, Rand T. Efficient radiological assessment of the internal snapping hip syndrome. Eur Radiol 2001; 11:1743-1747.

6. Bae DK, Kwon OS. Snapping knee caused by the gracilis and semitendinosus tendon: a case report. Bull Hosp Jt Dis 1997; 56:177-179.

7. Coonrad RW, Spinner RJ. Snapping brachialis tendon associated with median neuropathy: a case report. J Bone Joint Surg Am 1995; 77:1891-1893.
8. Lyu SR, Wu JJ. Snapping syndrome caused by the semitendinous tendon: a case report. J Bone Joint Surg Am 1989; 71:303-305.

9. Kissenberth MJ, Wilckens JH. The snapping biceps femoris tendon: a case report. Am J Knee Surg 2000; 13:25-28.

10. Weskott HP. B-flow: a new method for detecting blood flow. Ultraschall Med 2000; 21:59-65.

11. Rubin JM, Bude RO, Carson PL, Bree RL, Adler RS. Power Doppler US: a potentially useful alternative to mean frequency-based color Doppler US. Radiology $1994 ; 190: 853-856$. 